\section{Ética de la asistencia sanitaria del adulto mayor en Ecuador}

Ortiz, José

Universidad de Cuenca.E-mail: jose.ortiz@ucuencaedu.ec. Freire, Ulises

Universidad de Cuenca

Ortiz, Pedro

Universidad de Cuenca

PALAVRAS-CHAVE: Bioética, Adultos mayores, Asistencia sanitaria

Introducción Los Principios de la Naciones Unidas en favor de los adultos mayores (AM) son: independencia, participación, contar con los cuidados necesarios, autorrealización, dignidad (ONU, 1991); en este marco, la Constitución Política del Ecuador (Asamblea Constituyente, 1998) contiene normas de protección que se traducen en igualdad de derechos y garantías a favor de todos, ecuatorianos y extranjeros, independientemente de su edad. A partir del año 1984 el Ministerio de Bienestar Social, establece programas y proyectos en beneficio de los AM (MBS, 2014) y en el año 1991 se crea la Ley Especial del Anciano, según la cual el Estado garantiza el derecho a un nivel de vida que asegure la salud corporal y psicológica, la alimentación, el vestido, vivienda, asistencia médica, la atención geriátrica y gerontológica integral y los servicios sociales necesarios para una vida digna (Congreso Nacional, 2006). No obstante lo antes mencionado, en la práctica dicho grupo etario reclama mayor atención; de ahí que el objetivo del presente estudio es valorar en qué medida las acciones que emprenden las instituciones estatales consiguen satisfacción en los AM, que se evidencia mediante la percepción en el ejercicio de sus derechos y la calidad de los servicios de salud. Método Se realizó un estudio transversal que incluyó, de manera aleatoria a 557 adultos mayores residentes en áreas de cobertura de centros de primer nivel de atención, mediante la aplicación de un formulario validado, previa la aplicación del consentimiento informado por parte de estudiantes de la carrera de medicina, que recibieron capacitación y supervisión de docentes. Entre las variables analizadas estuvieron las características sociodemográficas, derechos del adulto mayor y percepción sobre la calidad de la atención de salud. Resultados La edad promedio de los participantes fue de $76 \pm 8$ años, la mayoría fueron mujeres, casadas, con educación primaria y un $22 \%$ de analfabetismo. Con respecto al ejercicio de los derechos, la percepción sobre estándares de vida como alimentación, vivienda, vestimenta, asistencia y seguridad social, no discriminación, salud, trato con dignidad, protección, participación comunitaria, varían entre $50 \%$ al $93 \%$; la edad, estado civil, ocupación, educación, se encuentran asociados, pero no en forma significativa; en tanto que el hecho de vivir solo está asociado de manera estadísticamente significativa (OR 2.31; IC 95\% 1.46-3.68) con el ejercicio de los derechos. En lo referente a la calidad de la atención, los adultos mayores califican como satisfactoria la infraestructura, fiabilidad en la atención, capacidad de respuesta del servicio, seguridad en el servicio, empatía con el personal, entre un 50 a $63 \%$. Conclusiones La insatisfacción percibida por los AM, tanto en el ejercicio de sus derechos, que está asociado con la soledad; como con la incompleta calidad en la atención de la salud, constituyen aspectos que impiden una vida digna. Esta situación da cuenta del incumplimiento de los principios éticos de beneficencia y justicia, lo cual genera la necesidad de conformar redes sociales de apoyo y coordinación interinstitucional.

AGRADECIMENTOS. Agradecemos a los docentes y estudiantes del Ciclo Comunitario del Internado Rotativo de la Carrera de Medicina.

\section{REFERÊNCIAS}

[1] Organización de las Naciones Unidas (ONU). Principios de las naciones unidas en favor de las personas de edad (1991).

[2] Asamblea Constituyente. Constitución de la República del Ecuador (1998).

[3] Ministerio de Inclusión Económica y Social. Norma Técnica Población Adulta Mayor Centros y Servicios Gerontológicos. Servicios y programas. Quito-Ecuador, (2014).

[4] Congreso Nacional del Ecuador. Codificación de la Ley del Anciano. Quito-Ecuador (2006). 